\title{
Consideraciones acerca de las cualidades del buen médico y del profesionalismo en medicina
}

\author{
Considerations on the characteristics of a good \\ physician and on professionalism in medicine.
}

\author{
Álvaro Toro • MEDELLín
}

¿Qué es un buen médico? Para decirlo en forma bien sencilla, es aquel médico que queremos para nosotros o para nuestros seres queridos en caso de una enfermedad.

Y ¿qué es profesionalismo en medicina? En un sentido estricto y purista sólo se consideran como profesiones cuatro ocupaciones: la medicina, la abogacía, el ministerio religioso y la carrera militar. Ellas se caracterizan por un estudio prolongado y muy especializado y porque en el momento de recibir la autorización para ejercerlas la persona se compromete, jura solemne y públicamente, a colocar el bienestar de aquellos a quienes sirve por encima de su propio bienestar. (Profesión viene de la palabra latina "profiteri", que significa comprometerse públicamente). Este compromiso público las distingue de otras ocupaciones y según los eticistas crea un contrato tácito con la sociedad y un compromiso solemne con aquellos a quienes se sirve.

La palabra "profesión" en relación con la medicina se usó por primera vez en el año 47 D.C. en un libro de recetas escrito por Scribonius, un médico romano de la corte del emperador Claudio y la define como la dedicación a la compasión en el alivio del sufrimiento (1).

Cuando busqué literatura sobre estos temas en la web me sorprendió la inmensa cantidad de artículos que se publican, de sitios tan diferentes como EE.UU., Inglaterra, Canadá, Francia, Suecia, Australia, Singapur, Hong-Kong, Israel, Nueva Zelanda, Finlandia, Sur África, España; encontré noticias de conferencias sobre la materia, libros, fundaciones dedicadas a su estudio y difusión. Esto es indicación del interés y preocupación que esto despierta.

Y ¿por qué razón? El progreso de la ciencia médica es inmenso, vertiginoso. Contamos hoy con medios técnicos y de tratamiento que nadie habría soñado hace pocos años. Tenemos medicamentos maravillosos que curan o alivian enfermedades hasta hace poco fatales. Los progresos de la cirugía son fantásticos. Pero hay otra cara de la moneda: el descontento del público con el cuidado médico que recibe va en aumento, la confianza que se le tenía al médico y su prestigio han caído a su nivel más bajo; las demandas contra él son cada día mas frecuentes; la medicina es cada momento más fría, más despersonalizada, menos humana. Estamos perdiendo esa hermosa y satisfactoria tradición altruista y filantrópica que viene desde Hipócrates y que pasaba de una generación médica a la siguiente.

Pero esto ¿qué importa si la medicina es cada día más satisfactoria y logra mejores resultados? ¡Sí importa y muchísimo! Empecemos por notar que siempre hay y habrá enfermedades crónicas e incurables con su carga de sufrimiento. Y toda enfermedad es una tragedia que le sucede a un ser humano en un momento puntual de su vida y tiene implicaciones que van muchísimo mas allá de un simple fenómeno biológico. Conlleva elementos de incertidumbre, temor, sufrimiento físico, pérdida de seguridad y autonomía. Variables de una persona a otra según nuestro temperamento, educación, sentimientos religiosos, momento en que nos encontremos de la vida, obligaciones, etc, pero siempre presentes. Por ésto, para cumplir a cabalidad el papel de médico no basta con tener el conocimiento científico para enfrentar la enfermedad; hay que tener los conocimientos, la habilidad y la disposición para prestar una ayuda integral al enfermo que sufre.
Dr. Álvaro Toro Mejía: Internista, Clínica Soma, Medellín.

Correspondencia: Dr. Álvaro Toro Mejía. E-mail: alvarotoro@une.net.co

Recibido: 29/VIII/2010 Aceptado: 28/X/2010 
Una medicina científicamente competente pero sin un componente humano no es suficiente para ayudarle a un paciente a enfrentar la pérdida de la salud y a encontrar el significado del sufrimiento. Los enfermos necesitan médicos que comprendan su enfermedad, que traten sus problemas médicos pero que también les extiendan su empatía y los acompañen honesta y valerosamente en su angustia. Anatole Broyard, un ensayista y editor del New York Times, cuando se encontró enfrentado a un carcinoma de próstata con múltiples metástasis escribió muy elocuentemente:

"Para los médicos mi enfermedad es sólo una rutina en sus rondas diarias, pero para mí es una terrible crisis en mi vida. Me sentiría mucho mejor si tuviera un doctor que comprendiera ésto... desearía... que siquiera una vez, por un momento, se uniera a mí, investigara mi espíritu, mi alma, así como estudia mi próstata; que entendiera que cada ser humano se enferma de una manera particular. Sin esta ayuda me siento que no soy nada... soy sólo una enfermedad" (2). "La enfermedad separa a las personas del mundo que conocieron... mi médico debería imaginarse la soledad de quien está gravemente enfermo; una soledad espantosa... no todo paciente puede ser curado; pero su sufrimiento sí puede ser aliviado por la manera cómo el doctor interactúa con él y en ésto el médico se está salvando a sí mismo. Debe comprender que su silencio y su neutralidad son innaturales, debe renunciar un poco a su autoridad y cambiarla por su humanidad... tiene poco que perder y mucho que ganar recibiendo al enfermo en su corazón".

Por ésto la pregunta frecuente: Usted ¿qué prefiere: un médico técnicamente muy competente o un médico muy humano? Es una pregunta tonta. Tan malo es un médico deficiente en conocimientos, así sea muy humano, como uno que sea muy sabio que no sea muy humano. Un médico digno de este nombre requiere ambas cualidades (3).

Muchos grandes de nuestra profesión han expresado estas ideas maravillosamente. Francis Peabody, el gran clínico de Harvard y del Johns Hopkins, en su elegante ensayo: "La atención del enfermo", publicado en 1927, lo dijo en palabras que tienen igual vigencia hoy:

"El tratamiento de la enfermedad puede ser completamente impersonal; el tratamiento del paciente debe ser totalmente personal ... lo que se llama un cuadro clínico no es una fotografía de un hombre enfermo en su cama; es una pintura impresionista del paciente, rodeado de su hogar, su trabajo, sus relaciones, sus amigos, sus alegrías, sus tristezas, esperanzas y temores. El buen médico debe dispensar generosamente tiempo, simpatía y comprensión. Una de sus características esenciales es su interés en lo humano, porque el secreto del cuidado del enfermo está en el genuino deseo de ayudar a ese ser humano que sufre" (4).
William Boyd, el patólogo canadiense, autor del libro de anatomía patológica que usaba como texto nuestro inolvidable maestro, el Dr. Alfredo Correa Henao, escribió:

"El paciente con una enfermedad cardiaca no es simplemente un motor de combustión interna con una válvula que deja escurrir; sino un ser humano con los mismos sentimientos que todos tenemos y con un corazón enfermo... con frecuencia es el hombre o la mujer y no simplemente una enfermedad lo primero que debemos tratar" (5).

Esta dualidad del médico, por un lado como científico y por otro como humanista que comprende, comparte y se esfuerza por consolar a su enfermo ha sido entendida desde tiempos muy antiguos y sigue siendo resaltada por notables clínicos y filósofos de la medicina a la hora actual.

Por ejemplo: todos conocemos aquella frase anónima, originada en Francia en el siglo XV acerca de los fines que debe buscar el médico: "Guerir quelque fois, soulager souvent, consoler toujours", (Curar a veces, aliviar frecuentemente, consolar siempre). Herrick, el clínico de Chicago, a quien debemos la descripción del infarto cardiaco, dijo:

"El verdadero médico debe poseer una personalidad doble; la del científico respecto a la enfermedad y la humana y compasiva respecto al paciente" (6).

Sir John Lewis, el padre de la comprensión de las arritmias, escribió:

"Es deber ineludible del médico para con su enfermo explicarle en forma cuidadosa y compasiva, aliviar su sufrimiento, disipar sus temores, animarlo, aconsejarlo,...no olvidemos que para él lo más importante del día es la visita de su médico, no la hagamos ruda ni apresurada" (7).

Mi mentor espiritual, William Osler:

"La práctica de la medicina es un arte, no un oficio, una vocación no un trabajo; una vocación en la cual su corazón tiene que ejercitarse tanto como su cabeza" (8).

Y por último Philip Manning en su libro "Manteniendo la pasión":

"Las características de los clínicos prominentes que han preservado la pasión por la medicina siempre han sido: una mente inquisitiva, analítica, una sed insaciable por nuevos conocimientos y una profunda compasión por los que sufren" (9).

Este aspecto humanístico de la medicina está hoy amenazado por múltiples factores:

La prestación de servicios médicos se convirtió en una industria con un potencial económico inmenso. Esto trajo cambios conceptuales enormes. El acto médico se convirtió en un producto sometido a las leyes del mercado, se compra, se vende y los hay de diferente calidad (lo mismo que hay televisores más o menos lujosos según se disponga de más o menos dinero para comprarlos). El paciente pasó a cliente, y hay buenos y malos clientes, (los malos son los pobres, los ancianos, los que tienen una "preexistencia"). 
El médico se transformó en proveedor, con horas de trabajo claramente limitadas, sin la unión, la responsabilidad y el conocimiento que antes tenía de su enfermo. Todo esto tiene profundas repercusiones éticas, que no es del caso discutir acá, el interesado puede encontrarlas en el excelente libro de Edmond Pellegrino (10). Digamos únicamente que hay una ligera diferencia entre ir a donde un distribuidor y decirle: "Quiero comprar un automóvil" e ir a donde un médico y decirle: "Doctor me asfixio cuando camino media cuadra y estoy tosiendo con sangre".

Esto ha traído elementos completamente nuevos, ajenos al principio que siempre, siempre, ha regido la profesión: "prima el bienestar del enfermo antes de toda otra consideración". En cambio ahora tenemos:

Burocratización: entre el paciente y el médico hay capas y capas de administradores: del gobierno, de las medicinas prepagadas, de los centros hospitalarios; que guiadas por un norte de rendimiento económico imponen reglas y políticas que cortan la autonomía del médico, frecuentemente en contra del mejor interés del paciente limitando, por ejemplo, el tiempo que se le debe dedicar a cada consulta, el número y tipo de exámenes que se pueden pedir y los tratamientos que se pueden ordenar.

Industrialización: el principio básico de la industria es: aumentar la producción al menor costo posible, utilizando la mano de obra más barata que se logre conseguir y reemplazando el trabajo humano por medios tecnológicos. Esto a veces reduce el médico a un simple eslabón en una cadena de producción, un técnico que hace bien una pequeña actividad, pero lo quita la visión comprensiva, completa del enfermo.

Comercialización: buscando atraer el mayor número de "clientes" posibles y no olvidemos: los médicos no tenemos clientes, tenemos pacientes. Esto se hace con propagandas, no siempre éticas ni verídicas: "la EPS xx le permite libre escogencia de médico y de clínica" "Nuestro examen ejecutivo es el mejor porque tiene el mayor número de pruebas"; (pruebas que frecuentemente no tienen ningún respaldo científico de beneficio y son sólo otra maniobra para obtener dinero). Y las casas de fabricantes de drogas e implementos médicos, inundándolos con sus propagandas, con frecuencia no ciertas o muy sesgadas; un estudio en España mostró que el 44.5\% de la información proporcionada por visitadores a los médicos de familia era errónea o tendenciosa, en Argentina el porcentaje fue $46 \%$ y en Alemania $94 \%$ (11-14). Es ilustrativo leer el escándalo acerca de la promoción del neurontín en un número reciente del New England Journal of Medicine (15). Y nos dan regalos, comidas, cocteles, viajes y a veces hasta dinero por el uso de determinados productos, (lo cual es una flagrante violación de la ética médica), no hay que ser muy malicioso para adivinar de qué bolsillo sale el dinero para estas dádivas.

No olvidemos que la meta primera de un negocio es: "Obtener las mayores utilidades posibles para los dueños" (Milton Friedman) (10). Bien distinto de la ética del profe- sional médico: "Colocaré el bien de mi enfermo antes que el mío propio".

Naturalmente que frente a los altos costos del cuidado médico y ante la imperiosa necesidad de justicia social, que distribuya equitativamente los recursos de salud disponibles, son indispensables: las regulaciones del Estado; la acción de expertos en industria y en ciencias administrativas; los auditores; que, en colaboración con los clínicos, hagan: los procedimientos más eficientes y seguros; eviten los esfuerzos y gastos superfluos; hagan la atención más pronta; la cobertura de salud más amplia y más igualitaria; ayuden a evitar los errores. Pero esto debe ser en un trabajo conjunto con los médicos, en el cual cada uno debe aportar su área de experto y no en forma de reglas e imposiciones que a los últimos sólo les queda aceptar.

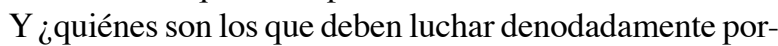
que esto se cumpla? En mi opinión las sociedades médicas; que hasta el momento han sido muy pasivas. Ellas no deben limitarse a la protección gremial: (que tal cirugía o tal consulta se pague a tanto; o que esta cirugía sólo la puede hacer el que tenga determinado título). La protección gremial es justa y está bien (inclusive debería ser mayor; por ejemplo con seguros de enfermedad, vejez, protección legal, etc.), pero el papel de las sociedades debería ir mucho más allá: luchando por preservar el profesionalismo médico, la autorregulación de la medicina, la ética, el bien de los enfermos, la excelencia en los servicios, la justicia social en medicina, la vigilancia en la educación médica, etc.

Generalizar es un error y quiero resaltar que hay administradores de servicios de salud y EPS, que son muy conscientes de los problemas que la industrialización del cuidado médico ha traído y que se esfuerzan con excelentes programas por evitarlos.

La fragmentaclón de la medicina dificulta gravemente la formación y cuidado de una buena relación médico-paciente que sea profunda y tenga valor terapéutico. Es muy frecuente y prácticamente la regla en pacientes mayores, el que tengan varias patologías: son hipertensos, diabéticos, han tenido infarto cardiaco, tienen artrosis, glaucoma y una próstata grande que debe ser operada; total son atendidos por cinco o seis médicos diferentes, que frecuentemente no se molestan en comunicarse entre ellos; ninguno tiene una visión integral del ser humano que trata, sino que se limita a su campo de experto; se convierten en técnicos hábiles de visión estrecha. Como dijo William Mayo en uno de sus aforismos: "Con gran conocimiento de medicina y poca sabiduría, llenos de ciencia y faltos en la comprensión del ser humano" (16).

Naturalmente no significa ésto, que dado el inmenso crecimiento de la ciencia, no sea indispensable la intervención de expertos en un campo limitado; pero siempre, siempre, debe haber alguien que comprenda integralmente al enfermo; que coordine y enlace a todos los actores del cuidado médico.

A esta dificultad se suma el frecuente cambio de médicos. Hoy el contrato de la EPS es con la clínica A y el paciente es atendido por el Dr. "Fulano"; dentro de un mes el contrato es 
con la clínica B y lo atiende el Dr. "Zutano"; en otro mes es con la clínica C y pasa el Dr. "Perano". De ésto se quejan amargamente los jenfermos!, y si se trata de una cirugía, el cirujano atiende el paciente por 15 días, máximo un mes y luego es chutado para continuar con otro médico. ¿Será esto suficiente cuando se trata de una cirugía grande? Yo opino que no. Con razón es frecuentísimo que uno pregunte: ¿Quién lo operó? y le respondan: "no sé". Ésto puede ser bueno desde el punto de vista industrial, pero pésimo desde el punto de vista médico.

¿Y la libre escogencia del médico? Tradicional e inclusive consagrada en Colombia por la Ley 23 de 1981 (título Il, artículo IV), evidentemente no existe.

Otro elemento en el deterioro del profesionalismo es el afán de dinero, tentación a la cual sucumben algunos colegas. Se olvidan del precepto de mi amigo Sir William Osler:

"La práctica de la medicina no es un negocio y nunca lo puede ser. Nuestros semejantes no pueden ser manejados como un negociante que maneja el maíz o el carbón" (17).

Esto lleva a muchos males: hacer la consulta muy corta y superficial, "porque las EPS pagan muy mal" (lo he oído); o corretear de un sitio a otro, para ganar más, imponiéndose más trabajo del que pueden hacer bien; o aquellos conflictos de intereses haciendo exámenes no claramente indicados, porque ellos o su institución tienen el equipo que hay que usar, o procedimientos no necesarios, etc. Y nuevamente traigo a Osler:

“...Si tenéis en vuestro corazón el deseo de las riquezas, tal vez las obtengáis, pero habréis traficado vuestros derechos a una noble herencia, pervertido el merecido título del médico de Amigo del Hombre $\mathrm{y}$ falseado las mejores tradiciones de un gremio antiguo y honorable" (18).

Y más problemas: el secreto profesional, que venía desde Hipócrates y que la ética médica siempre ha considerado sagrado (16); que está claramente estipulado en la ley colombiana, Ley 23 de 1981, (parágrafo 4 del artículo 19). ¡Éste sí que se acabó!; los médicos cuentan los nombres y diagnósticos de sus pacientes. Las EPS exigen toda clase de datos que deberían ser de reserva profesional para autorizar procedimientos y facturas de cobro. Hay que llenar los RIPS diariamente con nombre y diagnóstico y estos datos pasan por toda clase de manos que no tienen ninguna obligación de guardar el secreto. Y cuando un hospital o grupo médico practica un procedimiento novedoso, o en un paciente que tenga una enfermedad espantosamente espectacular, se publica en los periódicos, con el nombre, fotos y dirección del enfermo. Hasta en un aviso del periódico reciente se hacía propaganda a un procedimiento médico con los nombres de las personas que ya se lo habían efectuado.

$\mathrm{Si}$ a alguno le quedan dudas en relación con el deterioro del profesionalismo médico en Colombia, le recomiendo que lea los excelentes artículos de los Drs. José Félix Patiño, Ramón Córdoba,Adolfo-Vera Delgado y Jaime Borrero, que cito en la bibliografía (19-24).
Quiero dejar muy claro, que si bien estos vicios están muy extendidos, no se puede generalizar. Hay muchos, muchísimos médicos que son modelos de profesionalismo y ética, que honran la profesión. Dentro de ellos puedo contar los que me han atendido a mí y a mi familia en las enfermedades y a los cuales guardo gratitud imperecedera; tantísimos amigos que son un ejemplo para mí y muchos otros con los cuales tengo contacto por algún enfermo común y que impresionan por sus dotes de verdaderos médicos.

No es ningún consuelo, pero muchos de los males que enumeré son mundiales, de ahí nace la preocupación que se nota, universalmente, por retornar al profesionalismo. Como dice el Dr. Doukas de la Escuela de Medicina de la Universidad de Pennsylvania:

"La orgullosa tecnología y la ciencia nos han dado maravillosos métodos diagnósticos, como el electrocardiograma, resonancia magnética, tests genéticos, etc., y de tratamiento, como nutrición parenteral, quimioterapia, trasplantes, etc.. Pero en alguna parte del camino se nos perdió el genuino interés por los pacientes y las familias que los aman" (25).

Es importante anotar que en los años 2000-2001, y bajo los auspicios del American College of Physicians y la Universidad de Harvard, se reunió en EE.UU. un grupo multidisciplinario; formado por clínicos, eticistas, representantes de los pacientes y representantes de muchas entidades de seguros y medicinas prepagadas; para estudiar el impacto que el nuevo ambiente de prestación de servicios médicos tienen en el profesionalismo médico y en los derechos de los pacientes y publicar unas guías acerca de cómo mejorar lo que necesita corrección y poner muy en claro los deberes y derechos que todos los actores en este tema tienen (26). ¿No podremos hacer algo similar en Colombia?

En el 2002 se publicó simultáneamente en Lancet y en Annals of Internal Medicine la Carta Guía del Profesionalismo en Medicina en el Nuevo Milenio; el resultado de varios años de trabajo por líderes del American College of Physicians; la Federación Europea de Medicina Interna, la Fundación Americana para la Certificación en Medicina Interna y la Sociedad Americana de Medicina Interna (27). Posteriormente múltiples sociedades médicas, de otras especialidades y de muchos lugares del mundo se han adherido a esta Carta (27).

En el preámbulo hacen hincapié en que el profesionalismo es la base del contrato que tiene la medicina con la sociedad. En los Principios Fundamentales resaltan el principio de la primacía del bienestar del paciente; el principio de la autonomia del paclente y el principio de la justicia social (para promover la justicia en la distribución de los recursos de salud).

En las responsabilidades profesionales enumeran: dedicación a la competencia profesional. Esto es el esfuerzo continuado de toda la vida a mantener actualizados los conocimientos médicos, la habilidad clínica y la capacidad de trabajar en grupo para prestar un servicio de excelente calidad. 
Total honestidad con el paciente. Es decir impartir siempre al enfermo la información necesaria acerca de la enfermedad, el tratamiento y sus posibles complicaciones; otras opciones y si ocurre un efecto adverso hacérselo saber con absoluta franqueza.

Mantener siempre en absoluta confidencialidad la información que obtengamos acerca del paciente; el famoso secreto profesional.

Tener siempre las relaciones con los enfermos en un plano de respeto. La enfermedad hace a los seres humanos vulnerables, dependientes y débiles; el médico jamás debe abusar de ésto para aprovecharse de ellos en ninguna forma.

Dedicación a mejorar la calidad de los servicios. No sólo con su esfuerzo para mantener la competencia clínica, sino también trabajando con otros profesionales para reducir los errores médicos, aumentar la seguridad de los pacientes, optimizar los resultados de la atención y minimizar el gasto superfluo de recursos de salud.

Dedicación a mejorar el acceso a los servicios de salud. Esforzándose, individual y colectivamente (por los hospitales en donde trabajan y las asociaciones a las que pertenecen), para que los cuidados sean prestados igualitariamente sin diferencias por clase social, situación económica, de creencias o ninguna otra discriminación.

Dedicación a la distribución justa de unos recursos que son finitos. Los médicos deben proporcionar a sus pacientes la atención que les sea necesaria, pero en una forma que sea costoefectiva; deben evitar escrupulosamente exámenes y procedimientos que sean superfluos.

Dedicación al conocimiento científico y su uso adecuado. Parte muy importante del contrato de la medicina con la sociedad se basa en la integridad y uso apropiado del conocimiento científico y la tecnología. Es deber de los médicos mantener metas científicas elevadas; promover la investigación; crear nuevo conocimiento y asegurarse de que la ciencia y la tecnología sean bien empleadas.

Dedicación a hacerse dignos de la confianza de la sociedad por el manejo muy escrupuloso de los conflictos de intereses. Esto se refiere a uso de procedimientos que el propio médico hace y a los beneficios que pueda recibir de compañías farmacéuticas, o fabricantes de implementos médicos, o de seguros y esto se aplica tanto al médico individual como a los hospitales y asociaciones médicas.

Dedicación a la responsabilidad profesional. Deben colaborar entre sí para que la atención del enfermo sea de la mejor calidad; deben ser respetuosos el uno del otro; deben ser activos en el proceso de autorregulación (incluyendo medidas de disciplina y de remedio para los médicos que hayan fallado en sus calidades profesionales). Deben también intervenir en la definición de los requerimientos y organización de la educación de sus miembros actuales y futuros.

Hasta acá la Carta del Profesionalismo en el Nuevo Milenio. Muy ambiciosa, sí ¿Pero nos debemos contentar con menos? ¡NO!
Algunos clínicos y eticistas, considerando excelente esta Carta piensan que hace mucho énfasis en el aspecto de contrato y de obligaciones del médico: "debe hacer esto o aquello" y no resalta, lo que para ellos es más importante; las virtudes que en toda la historia de la medicina han pertenecido al buen médico y son la base de la ética basada en la virtud y garantía de un profesional que busca la excelencia. Virtudes en el sentido aristotélico: "Las características que hacen a una persona buena y la capacitan para hacer su trabajo bien" $(28,29)$.

Estas virtudes son muchas; las más importantes (maravillosamente tratadas por Pellegrino en sus excelentes artículos y libros) $(1,10,30)$ son:

Fidelidad a la confianza que nos deposita el paciente: siempre dignos de esa confianza.

Benevolencia: actuando siempre por el bien del enfermo y naturalmente evitándole todo mal.

Compasión: sintiendo, considerando, el sufrimiento del paciente.

Honestidad intelectual: que nos permite conocer cuando no sabemos algo y tener la humildad para reconocerlo

Valor: que nos impulsa a cumplir el deber así haya un peligro físico, por ejemplo de contagio y a ser siempre abogados del paciente, cuando nuestra conciencia nos lo indica; por ejemplo en un sistema de salud comercializado, ante representantes de este sistema o de un hospital; así haya peligro de represalias. Valor para no ceder jamás en nuestros principios morales cuando el Estado, o el paciente, o la familia, o quien sea, nos pide hacer un acto médico que consideramos amoral.

Prudencia, (lo que Aristóteles llamó fronesis) y que se puede denominar sabiduría práctica, que permite pesar juiciosamente los elementos que entran en una situación determinada para tomar la mejor decisión.

Para Pellegrino los responsables de la educación médica, los médicos clínicos y las organizaciones profesionales; tenemos todos, una parte de culpa en el deterioro del profesionalismo (1).

Y ¿se puede enseñar el profesionalismo? todos estos autores, clínicos y educadores, afirman que sí; primariamente en las escuelas de medicina; también muchísimo en las sociedades médicas y en los hospitales; pero se requiere un esfuerzo consciente y dedicado.

En 2006 la Clínica Mayo publicó los resultados de una interesante encuesta hecha entre 192 pacientes que habían recibido atención por 14 especialidades diferentes (31). En ella se preguntaba cuáles eran las mejores y las peores experiencias que habían tenido con sus médicos durante su tratamiento; esto con el propósito de prestar una mejor atención y basados en la premisa de que la mayoría de las empresas de servicio se preocupan por enseñar a sus empleados cómo interactuar con sus clientes y lo mismo hacen las escuelas de administración con sus estudiantes; es entonces lógico pensar que lo mismo deben hacer los médicos con sus pacientes. 
En forma muy constante aparecieron siete cualidades que los enfermos consideran como básicas en el médico ideal y al contrario, su carencia lo hacen desagradable. Éstas son:

Inspira confianza: no sólo en relación con sus conocimientos y experiencia sino también en su actitud tranquila ante el paciente y la familia y que no se molesta por las preguntas que se le hagan, no importa que tan disparatadas parezcan.

Establece empatía: interpreta correctamente las preocupaciones verbales y no verbales del paciente y vuelve sobre ellas. Se esfuerza por entender lo que está sintiendo física y emocionalmente. Mira a la cara. Habla amablemente y calmadamente.

Humano: muestra su deseo de ayudar, es compasivo, presta atención no sólo a la enfermedad sino a la situación total del paciente. Usa el contacto físico cuando es apropiado.

Personal: averigua acerca de la vida, intereses y problemas del enfermo. Trata amablemente a su familia.

Franco: explica claramente, sin afán, sin terminología médica y repite las explicaciones cuando es necesario y en forma amable.

Respetuoso: explica y pide excusas cuando hace esperar al paciente; lo escucha con atención y no lo interrumpe; solicita su opinión cuando es apropiado; respeta su modestia durante el examen físico.

Cuidadoso: no escatima el tiempo, las visitas y consultas; da las instrucciones por escrito; expresa francamente cuando tiene que estudiar algún aspecto del caso; consulta con otros médicos y mantiene contacto con los otros que participan en el tratamiento.

A esta lista yo añadiría: el buen médico es: educado en sus modales; sencillo, nunca arrogante; se preocupa por actuar de acuerdo con los otros médicos tratantes; hace un gran esfuerzo por tener una buena comunicación con su enfermo.

$\mathrm{Y}$ una vez que ha aceptado su cuidado se preocupa de ser fácil de encontrar por él.

Evidentemente la lucha por la excelencia en la profesión nos corresponde a nosotros. Como dice el Dr. José Félix Patiño en su extraordinario artículo En Busca del Hipócrates Moderno:

"Los médicos deben ser los líderes en todo lo pertinente a la atención de salud en el siglo XXI. $\mathrm{Su}$ responsabilidad primaria es la preservación del profesionalismo de la medicina en el contexto del nuevo contrato social y deben luchar por la moralidad del "mercado de salud". Corresponde a la profesión salvaguardiar los principios humanitarios y de compromiso social de la medicina y la excelencia de la educación médica".

Para terminar y resumiendo todo lo que antes he dicho hay una regla, que si uno la practica nunca se equivocará en la atención de un enfermo; una regla que los médicos debemos siempre tener presente; es: la regla de oro promulgada por Confusio en una forma negativa 500 años antes de nuestra era: "no hagas a otros lo que no quisieras que otros te hicie- ran a $t i$ ". Y en forma positiva por Jesucristo: "hazpor otros como quisieras que otros hicieran por ti”.

\section{Referencias}

1. Pellegrino ED. Professionalism, Profession and the Virtues of the Good Physician. Mt Sinai J Med 2002; 69: 378-384.

2. Broyard A. Intoxicated by my illness and other writings on life and death. Ballantine. New York, 1992

3. Cassell EJ. The Nature of Suffering and the Goals of Medicine. (second edition), página 72. Oxford University Press. New York 2004.

4. Peabody FW. The care of the patient. JAMA 1927; 88: 877-882.

5. Boyd W. A Text Book of Pathology. 7th. Edilion, página 5. Lea and Febiger. Philadelphia 1961.

6. Aciemo LJ. Historia de la Cardiología. Educación Médica Continuada. Colombia

7. Lewis T. Diseases of the Heart. $2^{\text {nd }}$. Edition. The McMillan Company. New York 1937.

8. Osler W. The Master Word in Medicine. Aequanimitas. The Blakiston Company. Filadelfia 1942.

9. Manning PR, DeBakey L. Medicine: Preserving the Passion. Springer Verlag New York. 1987

10. Pellegrino ED. The Philosophy of Medicine Reborn. Páginas 101.-126. Universily of Notre Dame Press. Notre Dame, Indiana. 2008.

11. Rivera Casares F, Richart Rufino MJ, Navas Cutanda J, Rodríguez Górriz E, Gómez Moruno C, Gómez García B. The scientific information that the pharmaceutical industry provides to the family doctors. Aten Primaria $2005 ; \mathbf{3 6}$ : 14-8.

12. Mejia R, Avalos A. Material impreso distribuido por los agentes de propaganda farmacéutica. Medicina 2001; 61: 315-8.

13. Tuffs A. Only $6 \%$ of drug advertising material is supported by evidence. Brit Med J 2004; 328 - 485 .

14. Blackmer J. Professionalism and the medical association. World Med J. 2007; 53: 58-73.

15. Lanfeld CS, Steinman MA. The nerontin legacy-Marketing through misinformation and manipulation. N Engl J Med 2009; 360: 103-6.

16. Mayo CH, Mayo WJ. Aphorisms. Mayo Clinic Dívision of Education and Resources, Rochesler Minn.

17. Osler W. On the Educational Value of the Medical Society. En: Osler Inspiration from a Great Physician. Bryan CS., Página 137. Oxford Unív. Press. New York 1997.

18. Osler W. El Maestro y el Estudiante. Aequanimitas. Página 42. The Blakiston Company. Filadelfia 1942

19. Patiño JF. La Desprofesionalización de la Medicina en Colombia. Acta Med Colomb. 2001; 26: 43-49.

20. Patiño JF. La desprofesionalización de la medicina en Colombia. Acta Med Colomb 2009; 34 (Supl.): 271-277.

21. Patiño JF. En busca del Hipócrates moderno. Persona y Bioélica 2004 (22-23) 2233.

22. Córdoba R. Deterioro de la relación médico-paciente y sus consecuencias en el ejercicio de la medicina. Anales de la Academia de Medicina de Medellín. 1999; 12: $45-50$.

23. Vera-Delgado A. Humanismo y medicina y algunas reflexiones pertinentes. Rev Colomb de Cardiol 2004; 11: 271-6.

24. Borrero J. Medicina y Humanismo. Páginas 159-164. Imprenta de la Univ. De Antioquia 2006. Medellin.

25. Doukas DJ. Returning to professionalism: The re-emergence of medicine's art. Am J Bioeth 2004; 4: 18-9.

26. Gail J. Povar, Helen Blumen, John Daniel, Suzanne Daub, Lois Evans, Richard P. Holm, Et al. Ethics in Practice: Managed care and the changing health care environment. Medicine as a profession managed care ethics working group statement. Ann Int Med 2004; 141: 131-6.

27. Blank L, Kimball H, McDonald W, Merino J; ABIM Foundation; ACP Foundation; European Federation of Internal Medicine. Medical professionalism in the new millenium: A Physician Charter 15 months later. Ann Int Med 2003; 138: $839-41$

28. Swick HM, Bryan CS, Longo LD. Beyond the physician charter: reflections on medical professionalism. Perspect Biol Med 2006; 42: 263-75.

29. MacKenzie CR. Professionalism and medicine. HSS J 2007; 3: 222-7.

30. Pellegrillo ED, Thomasma DC. The Virtues in Medical Practice. Oxford Univ Press, New York 1993.

31. Bendapudi NM, Berry LL, Frey KA, Parish JT, Rayburn WL. Patient's perspectives on ideal physician behavíors. Mayo Clin Proc 2006; 81: 338-44. 